\title{
Interpolating function of the strain relief of epitaxial quantum dots via an alternative morphological descriptor
}

\author{
Daniele Scopece* \\ Empa, Swiss Federal Laboratories for Materials Science and Technology, nanotech@surfaces Laboratory, Überlandstrasse 129, \\ CH-8600 Dübendorf, Switzerland and Department of Materials Science, University of Milano-Bicocca, 20126 Milano, Italy
}

(Received 19 November 2014; revised manuscript received 28 April 2015; published 26 May 2015)

\begin{abstract}
Assessing the equilibrium morphologies of self-assembled heteroepitaxial quantum dots requires the estimation of their elastic (volumetric), surface, and edge energy contribution, all of them being shape dependent. Due to the size and multifaceted morphology of these islands, the estimation of the first term is typically a time-consuming or complicated task. A general rule to predict it from the sole morphologies would guarantee a precious advantage in this field. Here we present an interpolating function to fulfill this purpose for the prototypical systems of $\mathrm{Ge} / \mathrm{Si}$ and InAs/GaAs. The trend is first extracted from a systematic analysis of realistic shapes observed on (001) substrates. It is then tested and corroborated for selected vicinal (tilted) substrates. Finally, the deviations due to intermixing and the underlying wetting layer are quantified. Of fundamental importance in this process is the identification of a morphological descriptor more accurate than the widely adopted aspect ratio, the limitations of which are discussed.
\end{abstract}

DOI: 10.1103/PhysRevB.91.195318

PACS number(s): 68.35.bg, 68.35.Gy, 81.05.Ea

\section{INTRODUCTION}

The bottom-up solution to create nanosized objects currently represents a widely explored technique to overcome the limitations of top-down nanolithography for materials with novel and tunable properties for new technological nodes. High-gain photosensors [1,2], devices with quantum optical properties [3], efficient thermoelectrics [4-6], high-speed transistors [7,8], and the efficient strain engineering of the recently introduced membranes $[9,10]$ represent just a few examples of the applications that are feasible. The properties of the final devices depend critically on the morphology of the nanostructures and on their evolution and/or stability during processing [11].

Prototypical semiconductor systems in this field are the self-assembled nanoislands (quantum dots) of $\mathrm{Ge} / \mathrm{Si}(001)$ [12-14] or InAs/GaAs(001) [15,16] deposited via molecular beam epitaxy.

The deposited material (epilayer) first creates a planar wetting layer (WL) that is subject to compressive strain due to the lattice mismatch with the substrate (equal to $\approx 4$ and $7 \%$ for $\mathrm{Ge} / \mathrm{Si}$ and InAs/GaAs, respectively). This strain is partially relieved by the system via the formation of three-dimensional (3D) structures (islands) with shallow facets atop the thin WL.

The higher strain relief guaranteed by steeper facets is one of the driving forces for the continuous morphological evolution of the islands [11,16-20]. In case the morphology observed is not kinetically -limited [21,22], as turns out to be the case in most standard deposition conditions [23], this morphological transition can be qualitatively [18] and also quantitatively $[16,19,24]$ explained in terms of a balance of elastic, surface, and edge energy (see Sec. II A for a review).

The surface energy contribution can play an important role in some subtle cases where a precise quantitative balance of the terms is needed [19,25-27]. But most commonly the sequence of the evolution observed experimentally agrees well

*daniele.scopece@empa.ch; daniele.scopece.science@gmail.com with the one predicted by considering the elastic energy term alone (governing the evolution from flatter to steeper islands), both on flat $[15,20]$ and on patterned substrates [8].

Therefore an accurate prediction of the elastic energy density $\rho$ of the system island plus substrate for various island morphologies represents a first indication of the relative stability of the structures considered [20].

This information can, in turn, be useful and precious for further processing or to predict the onset of line defects [28,29]. The mathematical models currently available to predict the $\rho$ [30-32] fail for shapes as steep as the ones observed experimentally and for the variety of the multifaceted, threedimensional systems. An order parameter defined from a geometrical intuition $[20,33,34]$ has been proven to provide a good sorting of strain relief - the aspect ratio (AR), defined as the ratio of the height of the island over the square root of the basal area:

$$
\mathrm{AR}=\frac{h}{\sqrt{B}} .
$$

No systematic analysis, however, can be found that probes its validity on a wide variety of morphologies.

In this work we tackle this issue by performing a systematic analysis on a large number of isolated, uncapped, and nondislocated quantum dots (islands) of various shapes and with the different facet orientations that are observed on (001) substrates (Sec. II B). We compute their elastic energy within the linear elasticity theory by a finite element (FE) approach (Sec. II C), that allows us to overcome the theoretical problems mentioned. The aim is to find an interpolating function that allows us to predict the elastic relief of an island by considering its sole morphology.

We follow the same path that has led to the corroboration of the AR in literature. First we focus on ideal situations: flat substrates, pure $100 \%$ Ge and $100 \%$ InAs islands, with no intermixing considered as a channel for strain relief, and with no WL under the islands, which would lead to nonsimilarities in the strain relaxation and size-dependent results $[25,26]$. 
After highlighting the limitations of the aspect ratio (Sec. III A) as a univocal descriptor, we propose an intuition-driven alternative (Sec. III B) that allows a narrower interpolating function of the elastic energy. A definitive comparison between this new parameter and the AR is made on vicinal substrates (Sec. III C), that additionally proves the broader validity of the interpolating function.

The deviations from this trend are then quantified by releasing the simplifying hypotheses on selected cases: an alloy analysis (Sec. III D) allows to estimate the variation expected for both homogeneous and nonhomogeneous intermixing and the presence of the WL (Sec. III E) is considered. This allows its safe usage in real situations of the interpolating functions deduced.

Additional information on the analysis and the technical details to reproduce the data are reported in Ref. [35].

\section{METHODS}

\section{A. Theoretical approach to predict the equilibrium morphologies}

For the sake of clarity and completeness, in this section we briefly review the method adopted in literature to predict the energetic stability of the islands.

By considering an island of volume $V$, exposed facets $S_{i}$, and a base of $B$, the energy is computed taking the WL as the reference for energy (here the edge energy is neglected):

$$
\Delta E=V\left(\rho_{\mathrm{isl}}-\rho_{\mathrm{WL}}\right)+\sum_{i} S_{i} \gamma_{i}-B \gamma_{\mathrm{WL}}
$$

The energies of different morphologies of islands (taken as Wulff construction) are compared at different volumes by assuming that they grow self-similarly. A convenient definition of adimensional geometric factors $\beta$ is introduced to make this equation explicitly dependent on $V$ :

$$
\beta_{i} \equiv S_{i} / V^{2 / 3}, \quad \beta_{\mathrm{cov}} \equiv B / V^{2 / 3},
$$

where the $\beta_{i}$ are said to be the "exposed" surfaces and the $\beta_{\mathrm{cov}}$ are the "covered" ones. The equation thus transforms into

$$
\Delta E=V\left(\rho_{\mathrm{isl}}-\rho_{\mathrm{WL}}\right)+V^{2 / 3}\left(\sum_{i} \beta_{i} \gamma_{i}-\beta_{\mathrm{cov}} \gamma_{\mathrm{WL}}\right) .
$$

The elastic term can be rewritten by focusing on the amount of strain of the WL that is relaxed by the island considered:

$$
V\left(\rho_{\mathrm{isl}}-\rho_{\mathrm{WL}}\right)=V \rho_{\mathrm{WL}} \frac{\rho_{\mathrm{isl}}-\rho_{\mathrm{WL}}}{\rho_{\mathrm{WL}}} \equiv V \rho_{\mathrm{WL}}(-F),
$$

where we call $-F$ the "relaxation factor," which is negative since $\rho_{\text {isl }}<\rho_{\mathrm{WL}}$.

In this work we seek for an expression of $F$ as a function of a morphological descriptor. Since the $\rho_{\mathrm{WL}}$ is known analytically [35], this expression allows to completely quantify Eq. (4) if estimations of the surface energies $\gamma$ are given or known.

Here it is noteworthy to remember that, differently to metals, the surface energy of strained semiconductors depends not only on the direction of the facets $i$ but also on their reconstructions [36] and the strain state of the surfaces [37]. The determination of the stability of the different reconstructions for different strain represents currently an open field of research $[26,37,38]$. Since the strain state of the facets on the islands assumes different values for different morphologies of the island itself, a precise quantification for fine effects of the surface energies is performed on fixed shapes and with a multiscale approach [39]. In case of InAs/GaAs the surface energy is also dependent on the chemical potential to be used $[40,41]$. It is important to consider that the surface energies here $(\gamma)$ have both the meaning and the dimension of an energy per unit area $\left(\mathrm{meV} / \AA^{2}\right)$. This is not to be confused in these particular systems with the quantities needed to build the Wulff shape (see discussion in the next section).

The reader here is provided with a fast instrument to sort and predict the volumetric contribution of the internal energy of the islands, that represents the first step in assessing Eq. (4).

\section{B. Creation of the morphologies}

In order to be predictive with experiments, in this work we focus on the islands showing the same family of facets as in experiments: $\{105\},\{113\}$, and $\{15323\}$ for $\mathrm{Ge} / \mathrm{Si}$ and $\{137\}$, $\{101\}$, and $\{111\}$ in InAs/GaAs [15,42].

In Wulff construction the morphologies are created by specifying the facets appearing and the distances of these planes from the origin of axes. What determines the morphologies is
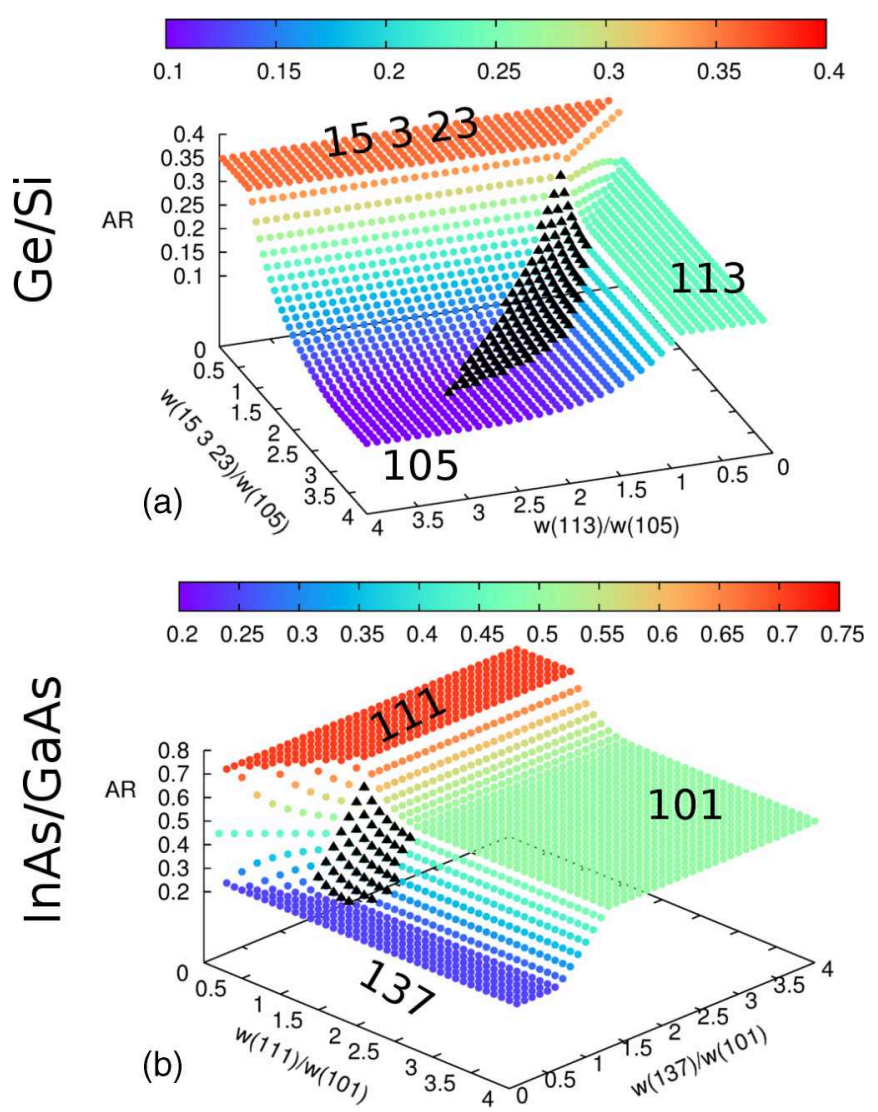

FIG. 1. (Color online) AR of the islands constructed by systematically varying the ratio of the surface energies $w$ in the Wulff construction. Every point corresponds to a morphology considered. The plateaus are labeled with the Miller indices of the facet of the pyramids generated. Black points show the cases where all the facets considered appear. See Sec. II B. 


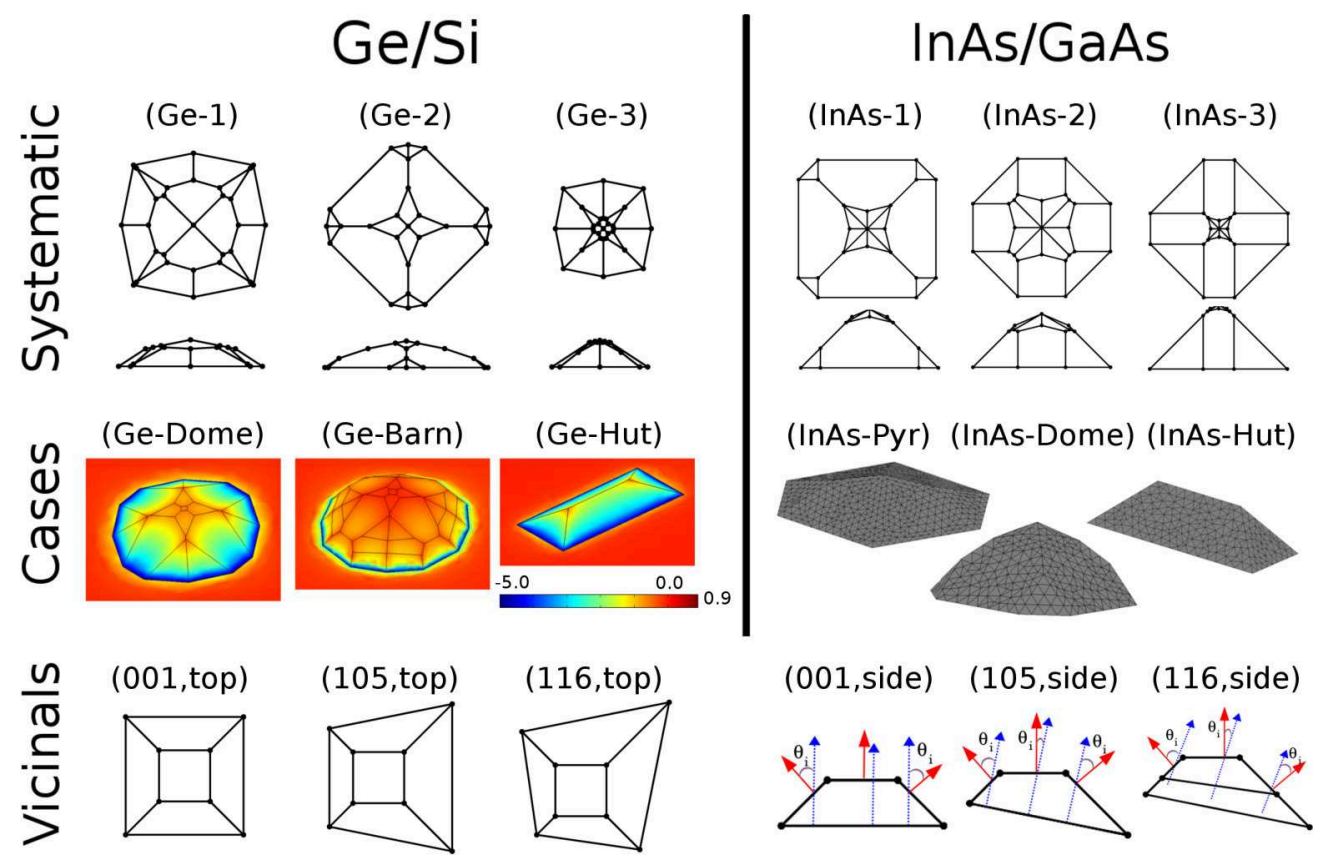

FIG. 2. (Color online) Morphology of some islands analyzed in this work selected from Fig. 1 ("Systematic"), from some cases added ("Cases") and the islands on different substrate orientation ("Vicinals"). The mesh in the islands, the strain component $\left(\epsilon_{x x}+\epsilon_{y y}\right) / 2$ (in $\left.\%\right)$, and the angles considered in the DAES are also shown.

the ratio between these distances, so here they are unitless. In this work we denote these distances as $w$. Although we stick to the convention of calling these distances "surface energies," they must not be confused with the $\gamma$ in the case of strained semiconductors (see the previous section).

In this work we vary these values $w$ to create the different morphologies and they have no energetic connotation. The morphologies are built with the Wulff construction code SOWOS [43], by systematically varying the "surface energy" values of each family $w$ between 1 and 50 with a step of 1. We call this method "systematic" and it leads to about 140 meshable distinct morphologies per situation, so to have statistics on similar morphologies. Every input file is reported in Ref. [35] for the sake of reproducibility.

In this case the values of the AR for the morphologies considered can be visualized on a $3 \mathrm{D}$ graph as a function of the ratios of the $w$ [Figs. 1(a) and 1(b)]. Every point in this graph represents an island's shape. Here just one family of facets (pyramids, represented as plateau regions in Fig. 1), two facets (colored regions), or three facets (black points) appear. A given value of the AR is obtained by more than one point in this graph, corresponding to different morphologies with relative extension of the facets. Examples of this are the islands denoted $(\mathrm{Ge}-1)$ and $(\mathrm{Ge}-2)$ in Fig. $2(\mathrm{AR} \approx 0.20)$ and (InAs-1) and (InAs-2) $(\mathrm{AR} \approx 0.43)$; see Table I. The situation would be even more complicated in the case of more families of facets considered.

The line "Cases" in Fig. 2 sketches additional islands considered. Pyramids (Pyr), Domes, and Barns [15,16,39,42,44] are the morphology experimentally observed. The elongated, one-dimensional islands (huts) are included for their interest in producing nanowires [19,24,27,45-48]. As representative examples of these elongated islands, here only the huts of $\{113\}$ for $\mathrm{Ge} / \mathrm{Si}$ and $\{101\}$ for InAs/GaAs are considered for the sake of the clarity of the results graphs. We define the lateral aspect ratio (LAR) of the hut as the ratio of the longest edge of the base over the shorter one. Intuitively, the higher the LAR, the higher the elastic energy. Here huts of LAR equal to 1.0 (pyramid), 1.5, 2.0, 3.0, 5.0, and 10.0 are considered.

The case "Vicinals" is described in Sec. III C. The morphologies are here described via the geometrical factors $\beta$ of Eq. (3), where the exposed surfaces are considered together:

$$
\beta_{\exp }=\sum_{i}^{\text {exposed }} \frac{S_{i}}{V^{2 / 3}} .
$$

They are computed directly in sowos [43]. Their trends are analyzed in Ref. [35].

\section{Finite element analysis}

The elastic relaxation is performed in COMSOL Multiphysics [49] within the linear elasticity theory by using

TABLE I. Properties of the "Systematic" cases depicted in Fig. 2. The "Island" column reports the name of the panel representing the island in that figure. Ge-1, Ge-2, InAs-1, and InAs-2 have a close value of the AR, but with very different morphology and $\beta$ values.

\begin{tabular}{lccccc}
\hline \hline Island & AR & $\beta_{\exp }$ & $\beta_{\text {cov }}$ & $F(\%)$ & DAES \\
\hline Ge-1 & 0.202 & 5.26 & 4.65 & 40.3 & 0.093 \\
Ge-2 & 0.203 & 5.82 & 5.28 & 35.9 & 0.078 \\
Ge-3 & 0.303 & 4.95 & 4.16 & 48.1 & 0.129 \\
InAs-1 & 0.436 & 4.50 & 3.22 & 57.1 & 0.215 \\
InAs-2 & 0.437 & 4.23 & 2.98 & 60.8 & 0.231 \\
InAs-3 & 0.557 & 4.26 & 2.76 & 64.2 & 0.273 \\
\hline \hline
\end{tabular}




\section{$\mathrm{Ge} / \mathrm{Si}$}
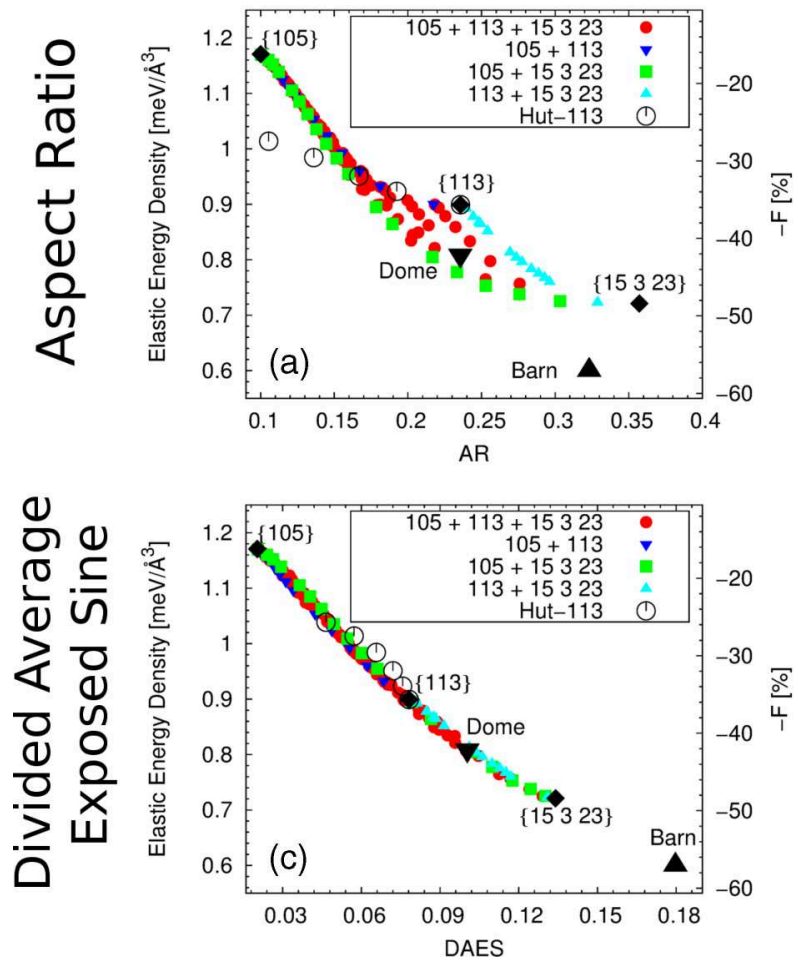

\section{InAs/GaAs}
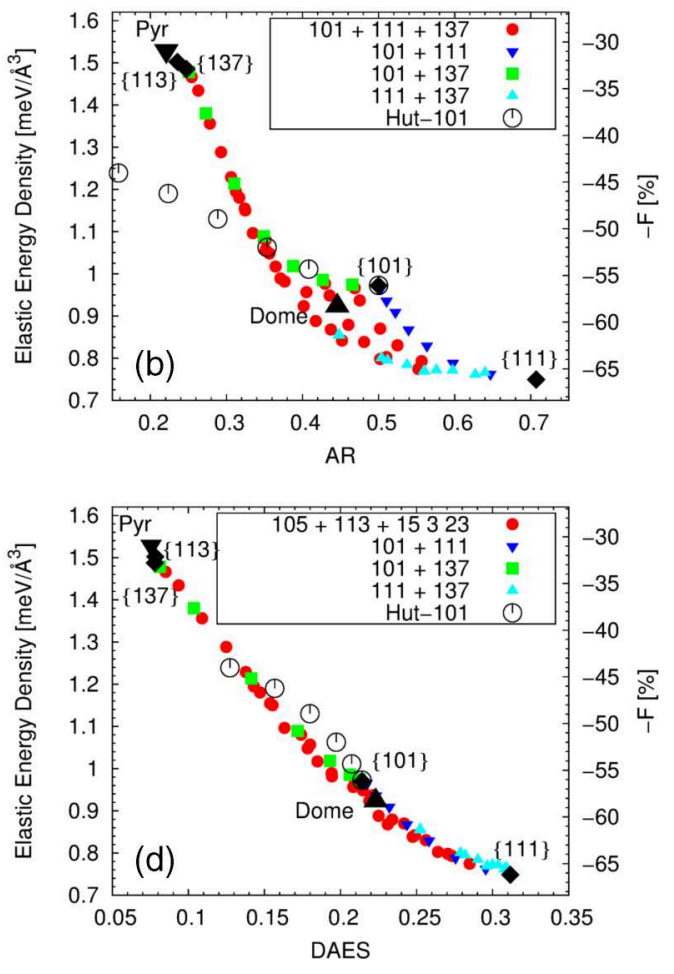

FIG. 3. (Color online) Elastic energy density $\rho_{\text {isl }}$ of the islands analyzed. The trend obtained with the parameter suggested in Eq. (8) (c), (d) is much narrower and more univocal than the one with the AR (a), (b).

the experimental elastic constants and the lattice mismatch reported in Ref. [35].

Although the real crystals are anisotropic, in some computational works isotropy is artificially imposed (i.e., $\left.C_{44}=\left(C_{11}-C_{12}\right) / 2\right)[29,50]$. We have checked whether this constraint alters the order found: it does not (not shown).

The islands are imported into COMSOL Multiphysics [49], expanded to a volume of $1000 \mathrm{~nm}^{3}$, and put on a flat surface of substrate ( $\mathrm{Si}$ or $\mathrm{GaAs}$ ). All the facets of the islands and the topmost surface of the substrate are left free to relieve strain, the bottom surface of the substrate is kept fixed in order to mimic a semi-infinite substrate, and to the lateral surfaces of the substrate periodic boundary conditions are applied.

With the aim at studying an isolated island excluding the effects of long-range strain field, the substrate size is taken more than 100 times the size of the islands $(2000 \times 2000$-nm wide in plane and 1000-nm thick). The same values of the elastic energy densities are obtained for a substrate ten times smaller (not shown), thus proving that it is large enough to avoid long-range effects.

A mesh is created by imposing a maximum mesh element of $3.0 \mathrm{~nm}^{3}$ in the islands (stressors). With this setting the number of mesh in the cells is $\approx 15000$ in every calculation, assuring the same level of accuracy in every case. The elastic energy obtained does not deviate considerably if a maximum element of mesh of $1.5 \mathrm{~nm}^{3}$ is adopted (not shown).
The elastic energy density $\rho_{\text {isl }}$ is defined as the total elastic energy stored in both the substrate and the island (stressor) per unit volume of the stressor and is expressed in $\mathrm{meV} / \AA^{3}$ :

$$
\rho_{\mathrm{isl}}=\frac{W_{\mathrm{isl}}+W_{\mathrm{sub}}}{V_{\mathrm{isl}}} .
$$

\section{RESULTS AND DISCUSSION}

\section{A. Aspect ratio}

The $\rho_{\text {isl }}$ obtained from the calculations are reported in Fig. 3 . On the right axes we report the relaxation factor $-F$ (in \%), defined in Eq. (5).

The $\rho_{\mathrm{WL}}$ obtained with the elastic constants adopted [35] are reported in Table II. Figures 3(a) and 3(b) report the data taking the AR as the morphology descriptor. For a chosen value of the AR various $F$ are possible depending on the geometries. Remarkable examples are the case of $\mathrm{AR} \approx 0.24$ for $\mathrm{Ge} / \mathrm{Si}$ and $\mathrm{AR} \approx 0.50$ for InAs/GaAs, where the distribution has its maximum width. The relief of a hut differs from the one of a faceted island (colored points) with the same AR considerably (the case of $\mathrm{LAR}=10.0$, leftmost point in the figure). The Barn in (a) lays out of the trend delimited by the other points.

Several points can be chosen by these graphs violating the rule "the higher the AR, the lower the $\rho$. ." This rule is only valid when a limited number of islands in a very narrow range of AR [33] or islands showing different families of facets are considered (e.g., pyramid, dome, and barn) [23,51]. However, 
these results prove that the AR is not general enough to define, sort, and, more importantly, predict the elastic relaxation of the islands from morphology (our aim in this work).

\section{B. Alternative descriptor}

It is known [17] that the islands relieve compressive strain by expanding through their facets in contact with a vacuum (exposed) and concomitantly compressing the substrate underneath. A facet would be more keen to expand the higher its steepness with respect to the substrate. Hence using an expression that represents an average estimation of the inclination of all exposed facets would help improve the description of the strain relief.

On the basis of this geometrical intuition, we define the quantity "divided averaged exposed sine" (DAES) (see Ref. [35] for a motivation of this choice):

$$
\mathrm{DAES}=\frac{\sum_{i}^{\text {exposed }} \beta_{i} \sin \left(\theta_{i}\right)}{\sum_{i}^{\text {exposed }} \beta_{i}} \times \frac{1}{\beta_{\mathrm{cov}}} .
$$

The angles $\theta_{i}$ are the ones between the normal of the exposed facet and the normal to the substrate (sketched in Fig. 2: "Vicinals").

The division by $\beta_{\mathrm{cov}}$ is introduced to properly describe the huts [35]. The sine is chosen so to have a higher value of DAES for higher relaxation expected and to have finite values for vertical walls [52].

The DAES makes the distribution of elastic energy much narrower and more univocal than the $\mathrm{AR}$ for both $\mathrm{Ge} / \mathrm{Si}$ and InAs/GaAs [Figs. 3(c) and 3(d)], approaching the ideal case of a one-to-one relation between elastic energy and morphological descriptor.

The trends for $\mathrm{Ge} / \mathrm{Si}$ and $\mathrm{InAs} / \mathrm{GaAs}$ are interpolated independently with the function

$$
-F(\%)=a_{0}+a_{1} \mathrm{DAES}+a_{2} \mathrm{DAES}^{2}+a_{3} \mathrm{DAES}^{3},
$$

whose fitting parameters $a$ are reported in Table III.

The fits overimposed on the entire set of data are shown in Fig. 4(a) as functions of their corresponding $F$ factors.

Some points of $\mathrm{Ge} / \mathrm{Si}$ are outliers $(\mathrm{DAES} \approx 0.21,0.80$, etc.) but a trend can be detected and the distribution around the best fit is quite narrow. The errors for InAs/GaAs are higher than for $\mathrm{Ge} / \mathrm{Si}$ because the distribution for the former is wider, but the trend is mostly respected. The wider distribution is

TABLE II. Properties and values of the "Vicinals" islands in Fig. 2. The order of $F$ is predicted by the DAES and not by the AR. See Sec. III C.

\begin{tabular}{lccc}
\hline \hline Substrate $\rightarrow$ & $(001)$ & $(105)$ & $(116)$ \\
\hline AR & 0.283 & 0.304 & 0.322 \\
DAES & 0.174 & 0.168 & 0.164 \\
$\rho_{\text {WL }}(\mathrm{Ge} / \mathrm{Si})\left(\mathrm{meV} / \AA^{3}\right)$ & 1.398 & 1.440 & 1.455 \\
$\rho_{\text {isl }}(\mathrm{Ge} / \mathrm{Si})\left(\mathrm{meV} / \AA^{3}\right)$ & 0.613 & 0.645 & 0.660 \\
$F(\mathrm{Ge} / \mathrm{Si})(\%)$ & 56.15 & 55.17 & 54.67 \\
$\rho_{\mathrm{WL}}(\mathrm{InAs} / \mathrm{GaAs})\left(\mathrm{meV}^{3} \AA^{3}\right)$ & 2.214 & 2.325 & 2.367 \\
$\rho_{\text {isl }}(\mathrm{InAs} / \mathrm{GaAs})\left(\mathrm{meV}^{3} \AA^{3}\right)$ & 1.047 & 1.110 & 1.137 \\
$F(\mathrm{InAs} / \mathrm{GaAs})(\%)$ & 52.70 & 52.28 & 51.95 \\
\hline \hline
\end{tabular}

TABLE III. Values of the best fit for Eq. (9) for $\mathrm{Ge} / \mathrm{Si}$ and InAs/GaAs reported in Fig. 3(f).

\begin{tabular}{lcc}
\hline \hline Parameter & $\mathrm{Ge} / \mathrm{Si}$ & InAs/GaAs \\
\hline$a_{0}$ & $-6.5 \pm 0.2$ & $-14.3 \pm 2.2$ \\
$a_{1}$ & $-465.2 \pm 6.5$ & $-239 \pm 40$ \\
$a_{2}$ & $1284 \pm 59$ & $75 \pm 216$ \\
$a_{3}$ & $-1377 \pm 136$ & $511 \pm 366$ \\
\hline \hline
\end{tabular}

due to the points of the huts (DAES $\approx 0.15-0.2$ ) deviating from the trend of the other islands. Another reason is the wide distribution of the $\beta$ s (influencing the strain relief) in terms of DAES for InAs/GaAs due, in turn, to some nonmonotonic trend with the family of facets considered [35].

In Fig. 4(b) the same trend by using the AR is shown for comparison: the drawbacks of this choice (nonunivocal, nonmonotonous, etc.) evidently rule it out as a good one.

\section{Vicinals}

The recent growing interest in the islands created on substrates other than (001) [19,24,26,32,53-58] compels to consider also this case. Here we use them not only as a case to test the generality of the interpolating function deduced
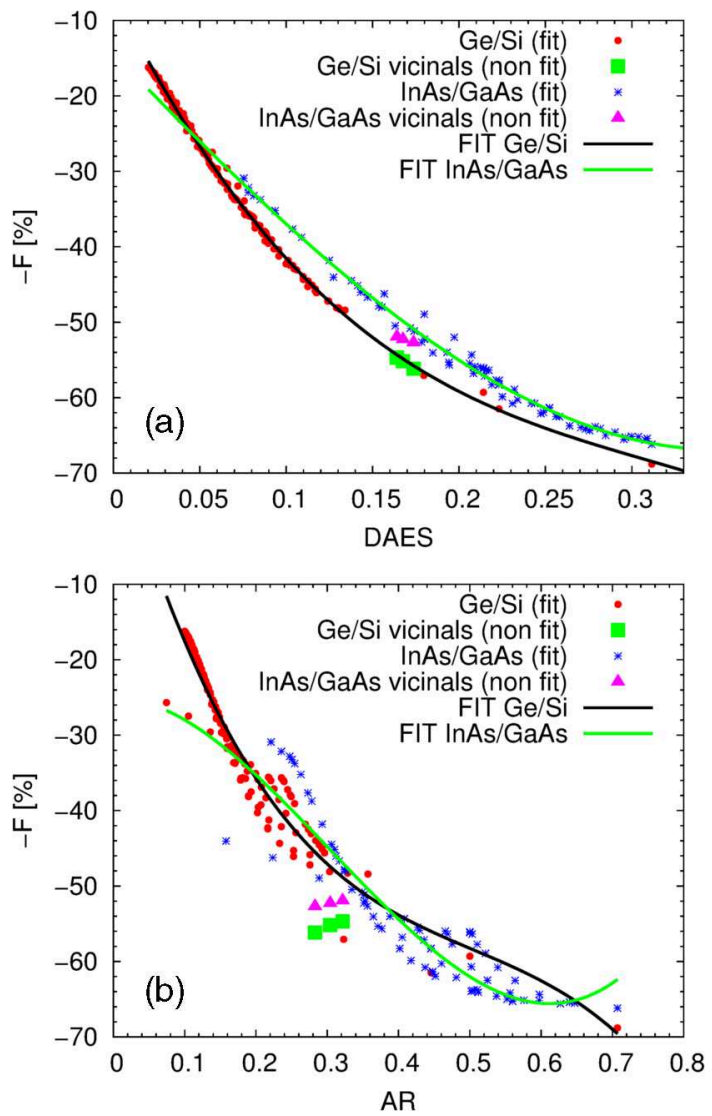

FIG. 4. (Color online) (a) Global fit with Eq. (9) for $\mathrm{Ge} / \mathrm{Si}$ and InAs/GaAs with the DAES describing the morphology. (b) The case of the AR for comparison. The improvement provided by the DAES and the limitations of the AR are clear. The points on the vicinals substrates fall on the trends predicted by using the DAES. 
but also as a validating example of the quality of this new descriptor over the standard AR. Indeed, we chose their morphology so to have an opposite ordering if classified via the AR or via the DAES (Table II and Fig. 2: "Vicinals"). The islands are created with the correct orientation of the basal area directly in sowOS [43], and are put in COMSOL on a flat substrate rotated along the vicinal surface considered.

The resulting elastic energy and morphologies are reported in Table II. In this case both the elastic energy $\rho_{\text {isl }}$ and the $F$ factor taken with respect to the substrate considered have an opposite trend from the one expected from the "AR-criterion": the islands relax less for the higher AR. In contrast, the trend is respected if the DAES is considered, even for the small difference of the DAES between the (105) and (116) $(0.1676$ versus 0.1641 ), computed from sowos [43] analytically. This supports our choice of the DAES as a proper morphology descriptor.

Although they are not included in the fit, these points fall on the one obtained from the DAES for the $\mathrm{Ge} / \mathrm{Si}$ or on the trend for InAs/GaAs [Fig. 4(a)]. This confirms the validity of the trend also for substrates other than (001) and that the interpolation deduced here can be conveniently used by experimentalists when handling islands whose DAESs fall into the range considered (spanning almost all islands normally observed experimentally). The same is not occurring in Fig. 4(b) for the AR.

\section{Alloys and nonhomogeneous concentration}

In this section we relieve the hypothesis of islands of pure Ge or InAs and analyze, for selected cases, the role of a different concentration homogeneous in the islands first and then discuss the effects of a nonhomogeneous distribution inside the island. In this case no WL is considered in the simulations.

In the simulations Vegard's law is applied for both the lattice constant of the stressor and its stiffness tensor [35]. We compute the elastic energy density $\rho_{\text {isl }}$ from FEM and use the $\rho_{\mathrm{WL}}$ of the corresponding concentration [35] in order to obtain the relaxation factor $F$.

Figure 5 shows the results for $\mathrm{Ge}_{x} \mathrm{Si}_{1-x} / \mathrm{Si}(001)$ (a) and $\mathrm{In}_{x} \mathrm{Ga}_{1-x} \mathrm{As} / \mathrm{GaAs}(001)$ (b) analyzed with a concentration between 100 and $20 \%$ of $\mathrm{Ge}$ and In, respectively. As a general trend the factor $F$ increases in modulus for more diluted systems. Its absolute variation from the pure cases in the case of $\mathrm{Ge} / \mathrm{Si}$ is between $2.5 \%$ (for the $\{105\}$ pyramid) and $3.5 \%$ (for the $\left\{\begin{array}{lll}15 & 323\end{array}\right\}$ pyramid). In the case of InAs/GaAs it is between $3.9 \%$ (for $\{111\}$ pyramid) and $5.6 \%$ (for the experimental pyramid "Pyr").

These values are the range of variation of $F$ to be expected on the trend reported in Sec. III B. In experiments the distribution of intermixed islands observed is typically nonhomogeneous $[8,44,59,60]$, but it has been proven that the three-dimensional concentration profile obtained is the one minimizing the elastic energy [23,61,62].

Reference [62] reports the degree of extra relaxation guaranteed by the nonhomogeneous distribution for the $\{105\}$ pyramid, dome, and barn of $\mathrm{Ge} / \mathrm{Si}(001)$. Using these data we can estimate the maximum deviation of $F$ expected for this system when a nonhomogeneous profile is present.
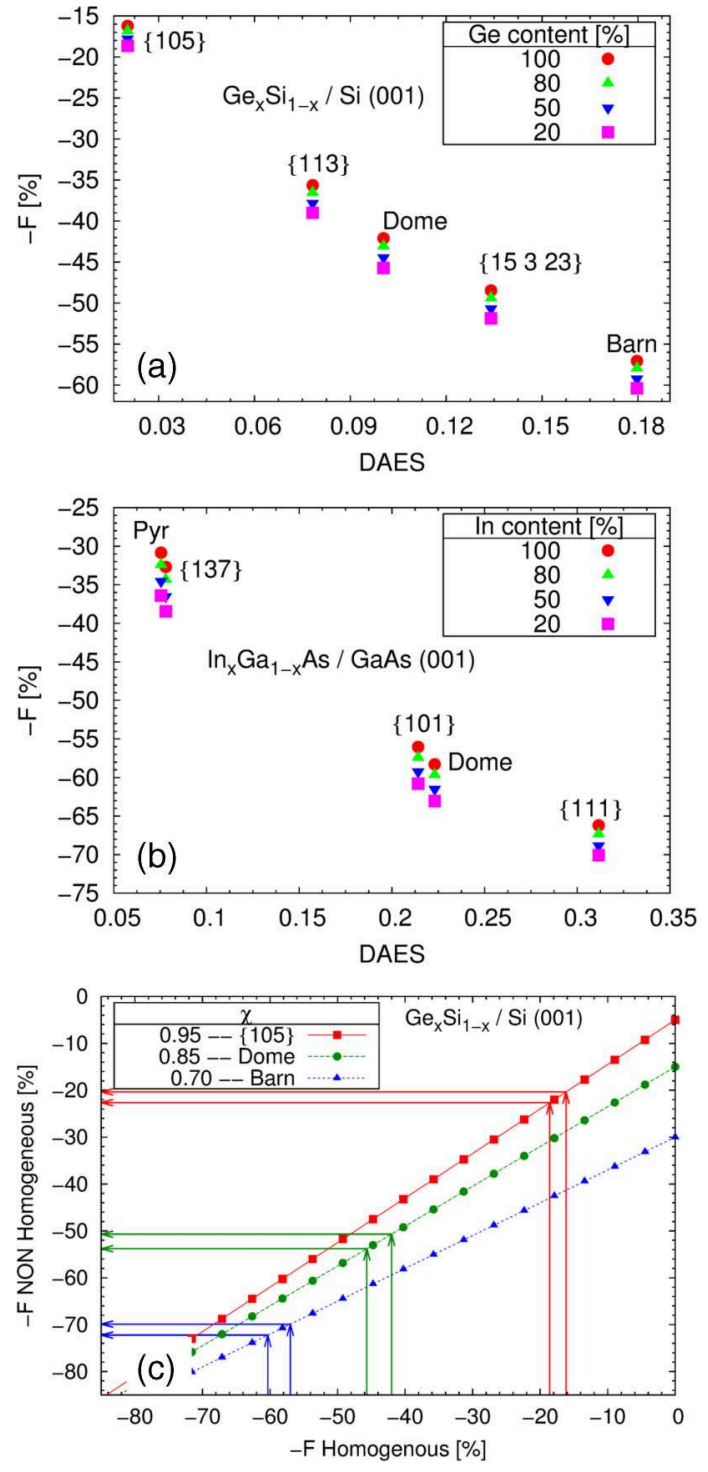

FIG. 5. (Color online) Deviation of the $F$ factor from alloying for selected islands on (a) $\mathrm{Ge}_{x} \mathrm{Si}_{1-x} / \mathrm{Si}(001)$ and (b) $\mathrm{In}_{x} \mathrm{Ga}_{1-x} \mathrm{As} / \mathrm{GaAs}(001)$. Panel (c) shows the expected $F$ factors in case of nonhomogeneous intermixing for islands of $\mathrm{Ge} / \mathrm{Si}$ that have been analyzed in literature (see discussion in Sec. III D).

By calling

$$
\frac{\rho_{\text {nonhomo }}}{\rho_{\text {homo }}}=\chi<1.0
$$

we can get

$$
-F_{\text {nonhomo }}[\%]=-F_{\text {homo }}[\%] \cdot \chi+100 \cdot(\chi-1)
$$

Figure 5(c) reports the trends with the corresponding values of $\chi$ deduced from Ref. [62]. The vertical arrows correspond to the maximum range of the $F$ determined in panel (a) of the same figure. The horizontal arrows show the expected maximum deviation for a nonhomogeneous intermixing. The deviation is larger for higher relaxation (modulus of $F$ ): $\approx 2 \%$ for pyramids, $\approx 8 \%$ for dome, and $\approx 10 \%$ for barns. These are the maximum corrections to be taken to the trend of Sec. III B, but it must be kept in mind that it decreases when average concentrations of $\geqslant 70 \%$ Ge are considered [62]. 

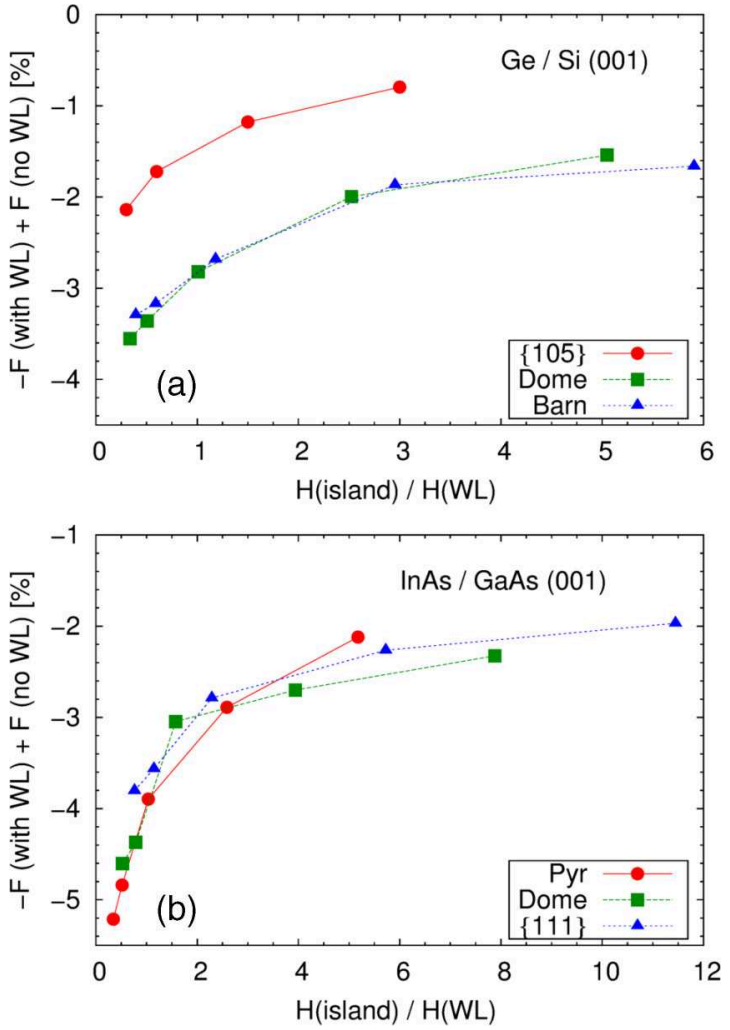

FIG. 6. (Color online) Deviation of the $F$ factor in the presence of a WL between the islands and the substrate. (a) Case of $\mathrm{Ge} / \mathrm{Si}(001)$. (b) Case of InAs/GaAs(001). The plots are reported as a function of the ratio of the height of the island over the height of the WL. The deviation is modest for the values of $H$ (island) $/ H(\mathrm{WL})$ in experiments (between 10 and 30). See Sec. III E for discussion.

To the best of our knowledge, no analysis is available in literature for the $\operatorname{In}_{x} \mathrm{Ga}_{1-x}$ As system.

\section{E. Wetting layer}

In experiments the islands have a residual, thin WL underneath during their evolution. Although the observed evolution is, at least qualitatively, compatible with simulations that neglect this thin WL, their elastic energy can be quantitatively altered. In this section we quantify the deviation of the elastic energy of the islands experimentally observed when they lay on a WL of the same concentration of various thicknesses. We fix the concentration of the islands to $100 \%$ Ge or InAs and the substrate is (001). In the calculations the WL is covering the substrate completely and the same periodic boundary conditions as the substrate are applied to its lateral borders.

We want to compare the elastic energy density of an island on the WL with the value of the same island of the same volume laying directly on the substrate. If we call $\rho_{\text {TOT }}$ the elastic energy density per unit volume of stressors (island plus WL) and $\rho_{\text {isl }}^{\prime}$ the elastic energy per unit volume of the island, the latter is computed as [35]

$$
\rho_{\mathrm{isl}}^{\prime}=\rho_{\mathrm{TOT}}+\frac{V_{\mathrm{WL}}}{V_{\mathrm{isl}}}\left(\rho_{\mathrm{TOT}}-\rho_{\mathrm{WL}}\right) .
$$

Figure 6 shows the deviation of the $F$ factor of the case with WL from the case of no WL. As expected from intuition, the deviation is larger the smaller the island, i.e., low value of the ratio of the height $H$ (isl) $/ H(\mathrm{WL})$. Typical values of this ratio for $\mathrm{Ge} / \mathrm{Si}$ cases can be extracted from Ref. [51], where the temperature is low enough to guarantee a low level of intermixing so to approach the case considered here for our analysis. By assuming the typical value of a four-monolayers thick WL $(\approx 0.5 \mathrm{~nm})$ the ratios are $\approx 10$ for the $\{105\}$ pyramids (typical volume of $5 \times 10^{3} \mathrm{~nm}^{3}$ ), $\approx 25$ for the domes (typical volume of $20 \times 10^{3} \mathrm{~nm}^{3}$ ), and $\approx 30$ for the barns (typical volume of $30 \times 10^{3} \mathrm{~nm}^{3}$ ). By extrapolation we can get that at these values the variation is within $1 \%$. For experiments at higher temperatures, the islands will be intermixed and have larger volumes, leading to an even smaller deviation.

The volumes for the InAs/GaAs can be taken from Ref. [15]. The ratio of the heights is $\approx 8$ for the pyramid (typical volume of $7 \times 10^{2} \mathrm{~nm}^{3}$ ) and $\approx 27$ for the domes (typical volume of $7 \times 10^{3} \mathrm{~nm}^{3}$, with facets steeper than the $\mathrm{Ge} / \mathrm{Si}$ case). No values could be found for the $\{111\}$ pyramid. Also in this case the difference is negligible by extrapolation.

\section{CONCLUSIONS}

In this work we have analyzed the elastic energy of a variety of islands' shapes systematically. Our aim was to extract an interpolating function in order to infer the elastic energy from the morphology of the islands. Our analysis has shown the limitations of the AR as a proper descriptor for this purpose and has led to the identification of a more accurate parameter (DAES). This choice has allowed to interpolate the data with a polynomial function. This function (and its deviations analyzed) can have both a theoretical and a practical valence.

From the practical point of view, it can be used to have an estimation of the relaxation factor $F$ of islands observed experimentally. The inclinations (normals to the facets) needed to compute the DAES can be extracted from experiments by using the method of the facet plot [63] and inserted in SowOS [43] to replicate the geometry.

In this way the DAES and the geometrical factors $\beta$ are deduced. From the DAES and our interpolation in Eq. (9), the value of $F$ can be extracted. From the $\beta$ and Eq. (4), the energetic curve can be plotted (if the surface energies $\gamma$ are known or estimated) for different shapes, so to predict the equilibrium morphologies to be expected among some possibilities.

From a merely theoretical perspective, the obtained trend could serve as a benchmark for future predictive theories of strain relaxation in complex morphologies.

\section{ACKNOWLEDGMENTS}

The author kindly acknowledges the three anonymous referees: their constructive criticisms have inspired and allowed considerable improvements over the original version. 
[1] H.-C. Chen, C.-W. Wang, S.-W. Lee, and L.-J. Chen, Advanced Materials 18, 367 (2006).

[2] C. Y. Chien, W. T. Lai, Y. J. Chang, C. C. Wang, M. H. Kuo, and P. W. Li, Nanoscale 6, 5303 (2014).

[3] H. Zhang, V. Kulkarni, E. Prodan, P. Nordlander, and A. O. Govorov, J. Phys. Chem. C 118, 16035 (2014).

[4] G. Schierning, Phys. Status Solidi A 211, 1235 (2014).

[5] P. Chen, J. J. Zhang, J. P. Feser, F. Pezzoli, O. Moutanabbir, S. Cecchi, G. Isella, T. Gemming, S. Baunack, G. Chen, O. G. Schmidt, and A. Rastelli, J. Appl. Phys. 115, 044312 (2014).

[6] H.-T. Chang, S.-Y. Wang, and S.-W. Lee, Nanoscale 6, 3593 (2014).

[7] L. Nanver, V. Jovanović, C. Biasotto, J. Moers, D. Grützmacher, J. Zhang, N. Hrauda, M. Stoffel, F. Pezzoli, O. Schmidt, L. Miglio, H. Kosina, A. Marzegalli, G. Vastola, G. Mussler, J. Stangl, G. Bauer, J. van der Cingel, and E. Bonera, Solid-State Electronics 60, 75 (2011).

[8] J. Zhang, A. Rastelli, O. G. Schmidt, and G. Bauer, Phys. Status Solidi B 249, 752 (2012).

[9] G. Vastola, V. B. Shenoy, and Y.-W. Zhang, ACS Nano 6, 3377 (2012).

[10] D. Scopece, F. Montalenti, M. Bollani, D. Chrastina, and E. Bonera, Semic. Sci. Techn. 29, 095012 (2014).

[11] J.-M. Baribeau, X. Wu, N. L. Rowell, and D. J. Lockwood, J. Phys.: Condens. Matter 18, R139 (2006).

[12] G. Medeiros-Ribeiro, A. M. Bratkovski, T. Kamins, D. A. A. Ohlberg, and R. S. Williams, Science 279, 353 (1998).

[13] J.-N. Aqua, I. Berbezier, L. Favre, T. Frisch, and A. Ronda, Physics Reports 522, 59 (2013).

[14] I. Berbezier and A. Ronda, Surf. Sci. Rep. 64, 47 (2009).

[15] G. Costantini, A. Rastelli, C. Manzano, P. Acosta-Diaz, G. Katsaros, R. Songmuang, O. G. Schmidt, H. V. Kanel, and K. Kern, J. Crys. Growth 278, 38 (2005).

[16] P. Kratzer, Q. K. K. Liu, P. Acosta-Diaz, C. Manzano, G. Costantini, R. Songmuang, A. Rastelli, O. G. Schmidt, and K. Kern, Phys. Rev. B 73, 205347 (2006).

[17] L. Miglio and F. Montalenti, in Silicon-Germanium (SiGe) Nanostructures: Production, Properties, and Applications in Electronics, edited by Y. Shiraki and N. Usami (Woodhead Publishing Limited, Cambridge, UK, 2011), Chap. 10, pp. 211-246.

[18] I. Daruka, J. Tersoff, and A.-L. Barabasi, Phys. Rev. Lett. 82, 2753 (1999).

[19] G. Chen, B. Sanduijav, D. Matei, G. Springholz, D. Scopece, M. J. Beck, F. Montalenti, and L. Miglio, Phys. Rev. Lett. 108, 055503 (2012).

[20] M. Stoffel, A. Rastelli, J. Tersoff, T. Merdzhanova, and O. G. Schmidt, Phys. Rev. B 74, 155326 (2006).

[21] G. Capellini, M. D. Seta, and F. Evangelisti, Mat. Sci. Eng. B 89, 184 (2002).

[22] G. Capellini, M. De Seta, F. Evangelisti, and C. Spinella, Mat. Sci. Eng. B 101, 106 (2003).

[23] A. Rastelli, M. Stoffel, J. Tersoff, G. S. Kar, and O. G. Schmidt, Phys. Rev. Lett. 95, 026103 (2005).

[24] B. Sanduijav, D. Scopece, D. Matei, G. Chen, F. Schaffler, L. Miglio, and G. Springholz, Phys. Rev. Lett. 109, 025505 (2012).

[25] M. Brehm, F. Montalenti, M. Grydlik, G. Vastola, H. Lichtenberger, N. Hrauda, M. J. Beck, T. Fromherz, F. Schäffler, L. Miglio, and G. Bauer, Phys. Rev. B 80, 205321 (2009).
[26] D. Scopece, F. Montalenti, and M. J. Beck, Phys. Rev. B 85, 085312 (2012).

[27] F. Montalenti, D. Scopece, and L. Miglio, Comptes Rendus Physique 14, 542 (2013).

[28] R. Gatti, A. Marzegalli, V. A. Zinovyev, F. Montalenti, and L. Miglio, Phys. Rev. B 78, 184104 (2008).

[29] F. Boioli, V. A. Zinovyev, R. Gatti, A. Marzegalli, F. Montalenti, M. Stoffel, T. Merdzhanova, L. Wang, F. Pezzoli, A. Rastelli, O. G. Schmidt, and L. Miglio, J. Appl. Phys. 110, 044310 (2011).

[30] J. Tersoff and R. M. Tromp, Phys. Rev. Lett. 70, 2782 (1993).

[31] V. A. Zinovyev, G. Vastola, F. Montalenti, and L. Miglio, Surf. Sci. 600, 4777 (2006).

[32] B. J. Spencer and J. Tersoff, Appl. Phys. Lett. 96, 073114 (2010).

[33] R. Gatti, F. Pezzoli, F. Boioli, F. Montalenti, and L. Miglio, J. Phys.: Condens. Matter 24, 104018 (2012).

[34] B. Voigtländer and A. Zinner, Appl. Phys. Lett. 63, 3055 (1993).

[35] See Supplemental Material at http://link.aps.org/supplemental/ 10.1103/PhysRevB.91.195318.

[36] P. Raiteri, D.B. Migas, L. Miglio, A. Rastelli, and H. von Känel, Phys. Rev. Lett. 88, 256103 (2002).

[37] P. Kratzer, E. Penev, and M. Scheffler, Appl. Surf. Sci. 216, 436 (2003).

[38] D. Migas, S. Cereda, F. Montalenti, and L. Miglio, Surf. Sci. 556, 121 (2004).

[39] D. Scopece and M. J. Beck, Phys. Rev. B 87, 155310 (2013).

[40] L. Wang, P. Kratzer, and M. Scheffler, Jpn. J. Appl. Phys. 39, 4298 (2000).

[41] K. A. Fichthorn, Y. Tiwary, T. Hammerschmidt, P. Kratzer, and M. Scheffler, Phys. Rev. B 83, 195328 (2011).

[42] G. Costantini, A. Rastelli, C. Manzano, R. Songmuang, O. G. Schmidt, and K. Kern, Appl. Phys. Lett. 85, 5673 (2004).

[43] D. Scopece, J. Appl. Cryst. 46, 811 (2013), https://sites.google.com/site/danielescopece84/softwareopen-source/sow os

[44] J. J. Zhang, F. Montalenti, A. Rastelli, N. Hrauda, D. Scopece, H. Groiss, J. Stangl, F. Pezzoli, F. Schäffler, O. G. Schmidt, L. Miglio, and G. Bauer, Phys. Rev. Lett. 105, 166102 (2010).

[45] I. Daruka, C. Grossauer, G. Springholz, and J. Tersoff, Phys. Rev. B 89, 235427 (2014).

[46] J. J. Zhang, G. Katsaros, F. Montalenti, D. Scopece, R. O. Rezaev, C. Mickel, B. Rellinghaus, L. Miglio, S. DeFranceschi, A. Rastelli, and O. G. Schmidt, Phys. Rev. Lett. 109, 085502 (2012).

[47] L. Persichetti, A. Sgarlata, M. Fanfoni, and A. Balzarotti, Phys. Rev. B 81, 113409 (2010).

[48] J. J. Zhang, A. Rastelli, O. G. Schmidt, D. Scopece, L. Miglio, and F. Montalenti, Appl. Phys. Lett. 103, 083109 (2013).

[49] COMSOL Multiphysics User's Guide manual, COMSOL, Inc., 2012, www.comsol.com.

[50] V. M. Kaganer, B. Jenichen, F. Schippan, W. Braun, and L. Daweritz, and K. H. Ploog, Phys. Rev. Lett. 85, 341 (2000).

[51] F. Montalenti, P. Raiteri, D.B. Migas, H. von Känel, A. Rastelli, C. Manzano, G. Costantini, U. Denker, O. G. Schmidt, K. Kern, and L. Miglio, Phys. Rev. Lett. 93, 216102 (2004).

[52] C. V. Falub, H. von Kanel, F. Isa, R. Bergamaschini, A. Marzegalli, D. Chrastina, G. Isella, E. Muller, Niedermann, and L. Miglio, Science 335, 1330 (2012).

[53] J. T. Robinson, A. Rastelli, O. G. Schmidt, and O. D. Dubon, Nanotechnology 20, 085708 (2009). 
[54] M. Xu, Y. Temko, T. Suzuki, and K. Jacobi, Surf. Sci. 576, 89 (2005).

[55] L. Du, D. Scopece, G. Springholz, F. Schäffler, and G. Chen, Phys. Rev. B 90, 075308 (2014).

[56] L. Persichetti, A. Sgarlata, M. Fanfoni, and A. Balzarotti, Thin Solid Films 543, 88 (2013).

[57] L. Persichetti, A. Sgarlata, M. Fanfoni, and A. Balzarotti, Phys. Rev. Lett. 106, 055503 (2011).

[58] L. Persichetti, A. Sgarlata, M. Fanfoni, and A. Balzarotti, Phys. Rev. Lett. 104, 036104 (2010).
[59] G. Chen, G. Vastola, J. J. Zhang, B. Sanduijav, G. Springholz, W. Jantsch, and F. Schaffler, Appl. Phys. Lett. 98, 023104 (2011).

[60] J. J. Zhang, A. Rastelli, O. G. Schmidt, and G. Bauer, Appl. Phys. Lett. 97, 203103 (2010).

[61] R. Gatti, F. Uhlik, and F. Montalenti, New Journal of Physics 10, 083039 (2008)

[62] D. Digiuni, R. Gatti, and F. Montalenti, Phys. Rev. B 80, 155436 (2009).

[63] A. Rastelli and H. von Känel, Surf. Sci. Lett. 515, L493 (2002). 